\title{
Double Auctions for Dynamic Spectrum Allocation
}

\author{
Wei Dong*, Swati Rallapalli*, Lili Qiu*, K. K. Ramakrishnan ${ }^{\dagger}$, Yin Zhang* \\ ${ }^{*}$ The University of Texas at Austin ${ }^{\dagger}$ Rutgers University
}

\begin{abstract}
Wireless spectrum is a precious resource and must be allocated and used efficiently. The conventional spectrum allocation lets a government (e.g., FCC) sell a given portion of spectrum to one provider. This is not only restrictive, but also limits spectrum reuse and may lead to significant under-utilization of spectrum. In this paper, we develop a novel truthful double auction scheme to let any resource owner (e.g., a cellular provider), who has spare spectrum at a given time, sell to one or more providers that need additional spectrum at that time. Spectrum auction is fundamentally different from conventional auction problems since spectrum can be re-used and competition pattern is complex due to wireless interference. We propose the first double auction design for spectrum allocation that explicitly decouples the buyer side and seller side auction design while achieving (i) truthfulness, (ii) individual rationality, and (iii) budget balance. To accurately capture wireless interference and support spectrum reuse, we partition the conflict graph so that buyers with strong direct and indirect interference are put into the same subgraph and buyers with no or weak interference are put into separate subgraphs and then compute pricing independently within each subgraph. We develop a merge scheme to combine spectrum allocation results from different subgraphs and resolve potential conflicts. Using conflict graphs generated from real cell tower locations, we extensively evaluate our approach and demonstrate that it achieves high efficiency, revenue, and utilization.
\end{abstract}

\section{INTRODUCTION}

Motivation: The explosive growth and dynamic nature of wireless traffic make it costly for a single wireless service provider to buy enough spectrum based on a long-term contract to sustain its peak load, which may only last for brief periods. This inefficiency of long-term spectrum allocation, prevalent today, motivates the need for dynamic spectrum access wherein a wireless service provider only obtains sufficient spectrum to support the "typical" traffic demands and can (i) purchase additional spectrum on-demand from other providers to satisfy higher traffic demands, or (ii) offer the spare spectrum to other providers for profit when there is lower demand.

While dynamic spectrum access is attractive and it is now technically feasible to dynamically change the spectrum to use on-the-fly [5], [19], [25], an important open issue remains how to allocate spectrum across multiple parties. It is essential to have an incentive framework that can effectively foster collaboration while guarding against dishonest behaviors.

Design objectives: We aim to achieve the following properties for the spectrum double auction, where the first three properties are necessary economic properties for a good double auction and the remaining three quantify the effectiveness of the auction: (i) Truthfulness: Bidders cannot benefit from bidding differently from their true valuation; (ii) Individual rationality: Bidders get non-negative utilities, i.e., sellers are paid no less 978-1-4799-3360-0/14/\$31.00 (C) 2014 IEEE than their asks and buyers do not pay more than their bids; (iii) Budget-balance: The total amount paid to the sellers is no more than the total amount received from the buyers. This prevents the auctioneer, who runs the auction, from losing money; (iv) Efficiency: It is the difference between the sum of the winning buyers' valuations and the sum of the winning sellers' valuations. To achieve good efficiency, the goods should be sold to the buyers that value them the most and be sold by the sellers that value them the least. [14] shows that it is impossible to simultaneously achieve truthfulness, budgetbalance, and maximum efficiency. Thus in this paper we aim to achieve truthfulness, budget-balancing, and high (but not maximum) efficiency. (v) Revenue: It is the total amount of payment from all winning buyers. A winning buyer may pay different amount from its bid, depending on the auction design. A higher revenue gives sellers stronger incentive to participate. (vi) Utilization: Unique to spectrum auctions, we seek to maximize the spectrum utilization by allowing as many buyers as possible to reuse the spectrum. We quantify the utilization based on the total number of buyers that are assigned spectrum.

Challenges: Designing a good double auction for spectrum reuse poses the following significant challenges. (i) How to accurately capture wireless interference among the buyers? This is necessary to support spectrum reuse, where multiple buyers may share the same spectrum. Achieving spectrum reuse is the key property that distinguish spectrum auction from traditional auction, which assumes all entities compete against each other for an item. (ii) How to design a truthful double auction? Simply applying truthful auctions for the sellers and for the buyers does not lead to a truthful double auction. It is critical to ensure participants cannot gain by manipulating the interaction between the two sides. This is especially challenging in a spectrum auction because the buyer side auction differs from the seller side in that we need to take into account wireless interference between buyers in order to support spectrum reuse. (iii) How to maximize the spectrum utilization while preserving budget balance? To maximize spectrum utilization, we need to sell as many channels as possible and let them be concurrently used by as many buyers as possible. However, this goal conflicts with budget balance, as selling more channels means a lower average price and a possibly lower revenue (as the price is determined by the loser's price). Lower revenue means that fewer channels may be sold due to the need to preserve budget balance. We will further elaborate these challenges in Section II-B.

Limitations of existing spectrum auctions: The advantages of double sided spectrum auctions have attracted lots of research attention. Despite considerable previous work, significant challenges remain. In particular, some works fail to support spectrum reuse while preserving truthfulness [20], [23]. The first work that satisfies all economic properties and supports spectrum reuse is TRUST [27]. It follows the classic McAfee's double auction [12], which jointly computes the auction result for buyers and sellers. However, to apply 
the classic design, it makes several simplifications that could sacrifice performance. Specifically, it randomly groups noninterfering buyers and requires buyers in the same group to win or lose together. So the fate of a group is determined by the lowest bidding buyer. A group can lose even if it has many high bidding buyers, which results in unfairness and low efficiency. It also enforces uniform pricing for all buyers that win the same channel, in which case the price can be no more than the lowest bid of these buyers. This limits total revenue and further hurts the auction performance because few channels can be traded in order to preserve budget balance. Several follow-up works share similar problems [24], [2].

Our approach. We develop a novel double auction for dynamic allocation of spectrum $\left(D A^{2}\right)$ in Section III. $D A^{2}$ uses separate designs of the buyer and seller side auctions while achieving truthfulness, budget balance, and individual rationality. Compared to the classic joint design strategy (i.e., McAfee [12]) used by most existing spectrum double auction designs [27], [24], a unique advantage of our separate auction designs for the buyers and sellers is that this enables flexible combinations of different buyer/seller side designs. This significantly increases the design space and can immediately benefit from future enhancement to auction design on either buyer or seller side. Moreover, it also allows different properties of both sides to be captured accurately, which is especially important for spectrum double auctions since the buyer side is much more complicated due to wireless interference. We show how to combine the design of two sides to ensure budget balance and identify the necessary properties of a single side auction in order for the double auction to be truthful.

$D A^{2}$ consists of three components: (i) seller side auction design, assuming $N$ channels sell, (ii) buyer side auction design, assuming $N$ channels sell, and (iii) determining the number of channels, $N$, to sell to satisfy budget balance.

Among them, the buyer side design is the most challenging component due to complicated wireless interference. We propose to partition the graph into subgraphs based on the wireless interference among the buyers. Graph partition enables us to compute allocation independently in each subgraph and then combine the results. Moreover, within each subgraph, in order to support frequency reuse, buyers are grouped into independent sets, where buyers in the same independent set do not interfere. The performance is sensitive to the independent set construction. The independent sets should be constructed in a bid-independent fashion to ensure truthfulness [27]. Graph partition reduces the randomness of independent set construction, and allows us to better capture the different competition patterns in each subgraph to achieve higher revenue by computing prices in each subgraph independently.

We identify important requirements og a good partition algorithm: cutting as few edges as possible while yielding balanced partitions. These two requirements correspond to the well known metric, called the Normalized Cut. We apply spectral clustering [15], which is an effective approximation algorithm to minimize the Normalized Cut. We then use the group bid definition in [24] to sort the groups and determine potential winners in a subgraph. We develop a novel pairwise merge procedure to combine the allocation results from subgraphs and preserve truthfulness. It merges the results from two subgraphs at a time and reduces the efficiency and revenue loss due to conflicts between subgraphs. In addition, we discuss practical issues of applying $D A^{2}$ in Section IV, such as incorporating quality of service, leveraging prior knowledge about bid distributions and how to avoid starvation.

We extensively evaluate our approaches using conflict graphs generated from real cell tower locations derived from a major US cellular provider (Section V). Our results show that $D A^{2}$ consistently yields high efficiency, revenue, and utilization, and out-performs the existing approaches by up to $62 \mathrm{x}$ in efficiency, $126 \mathrm{x}$ in revenue, and $65 \mathrm{x}$ in utilization.

\section{BACKGROUND}

\section{A. Spectrum double auction}

A double auction implements a double-sided market. The market consists of three types of entities: buyers, sellers and an auctioneer. Buyers submit bids which specify the item they are interested in and their maximum willingness to pay. Sellers submit asks which include the item they offer for sale and their asking price. The auctioneer evaluates the bids and asks and determines the winners and the items that are traded. The auctioneer also determines the amount to pay to the sellers and the amount to charge the buyers. All payments from buyers are paid to the auctioneer and the auctioneer pays the sellers. A buyer's utility is then the difference between his valuation of the item he wins and the amount he pays to the auctioneer. Similarly for the sellers.

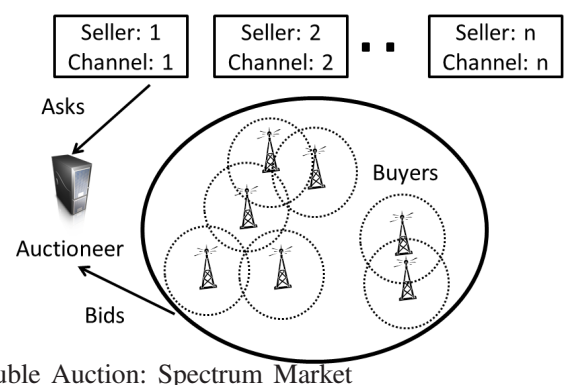

Fig. 1. Double Auction: Spectrum Market

Figure 1 shows a spectrum double auction. Here the sellers are spectrum resource owners (e.g., cellular service providers or organizations that own spectrum resource). The buyers can be any entities that need more spectrum. To capture interference relationships among the buyers, measurement can be conducted by the buyers, sellers, auctioneer, or a third party to derive the conflict graph. We can leverage existing approaches to either actively or passively measure the conflict graph (e.g., [17], [1], [18], [26]).

After winning the auction, buyers then start using the new spectrum by switching some of their clients onto the new spectrum. Techniques in intra-cell handover (e.g., [3], [4]) and spectrum virtualization (e.g., [5], [19]) can be applied to efficiently and seamlessly switch users to the new spectrum.

Basic auction settings: We consider a double-sided spectrum market where the goods of interest are wireless channels (frequency bands) and the players are spectrum resource users, such as cellular service providers. We assume a seller offers one channel for sale and a buyer seeks to buy one channel as in previous works [27], [24]. Multi-unit double spectrum auction is more challenging and is left for future work. A seller can sell 
to any buyer in the market. The auction runs periodically to enable spectrum reallocation in a dynamic fashion. The frequency of the auction depends on the volatility of demands and the cost of running an auction, which may include gathering inputs for the auction, computing the auction solution, disseminating auction outputs, and collecting payments.

Critical value is the lowest value that a buyer can bid and still win. Monotonic allocation means that a buyer who wins by bidding $x$ will still win if it bids $y$ where $y>x$. The definitions are similar for the seller side.

\section{B. Challenges in Spectrum Double Auction}

Challenges in supporting spectrum reuse: Spectrum auction is fundamentally different from a conventional auction in that an item in a conventional auction can be won by one buyer whereas spectrum can be reused by multiple buyers as long as they do not interfere with each other.

Wireless interference dictates how spectrum can be reused, so it has significant impact on the design of spectrum auction. Conflict Graph [8] is commonly used to capture the interference relationship between the buyers, where each node in the conflict graph denotes a buyer and there is an edge between two nodes if the corresponding buyers interfere.

Wireless interference has the following major impacts on the spectrum auction. First, conventional auctions do not allow an item to be bought by multiple buyers. However it is important to support spectrum reuse in spectrum auctions to maximize the utilization of the spectrum resource. Second, the conflict graph significantly complicates the competition among buyers. A buyer only directly competes with nearby interfering buyers, but at the same time a buyer's competition is coupled with other buyers' competition depending on their interference. Specifically, a buyer's auction outcome not only depends on its competitors' bids, but also depends on its competitors' competitors' bids and so on. This significantly complicates the auction design.

Challenges in designing a truthful double auction: As mentioned in Section I, it is challenging to achieve truthfulness in a double auction. Combining a truthful seller side auction and a truthful buyer side auction does not necessarily lead to a truthful double auction. This is because the single sided auctions do not need to consider budget balance, whereas in a double auction a participant may try to improve his utility by manipulating the point where budget balance is achieved and changing the number of traded items.

\section{Related works}

There are a few previous works on this topic. The pioneering and most representative work is TRUST [27] thus we focus on TRUST in our discussion. TRUST follows the classic McAfee's double auction design [12] to achieve the economic properties. To use McAfee's design, TRUST divides buyers into multiple independent sets or groups, where the buyers in each independent set do not interfere with each other. For each group, TRUST computes the group bid as the lowest bid in the group multiplied by the group size. Then McAfee is applied by treating each group as one virtual buyer. As in McAfee, all winning groups pay the group bid of the group in the sacrificed match. Within the group, all members share the price equally. TRUST enables spectrum reuse by grouping buyers, and achieves truthfulness by applying McAfee's design. It has several weaknesses:

- Low efficiency: The independent sets are constructed in a random fashion. It is possible that a high bidding buyer loses just because it is put into a bad independent set. As a result, the final winning groups can be suboptimal.

- Low revenue: The group bid only depends on the lowest bid in the group, and all higher bids are ignored. So the group payment is significantly limited by the lowest bid, which can be much lower than other bids in the group.

- Unfairness: TRUST uses uniform price for the buyers that are assigned the same channel. But buyers assigned the same channel may have different interference (competition) patterns. The uniform pricing forces some winners to pay for other winners' competition.

There have been several works on improving TRUST [22], [6], [24] and alleviating some problems in TRUST. However, they are still similar in spirit in that they still use random independent sets and enforce uniform price for buyers assigned the same channel, so they still suffer from low efficiency and poor fairness. For example, [22] improves on TRUST to sacrifice fewer buyers. However, it uses a single sided auction, a much simpler problem. [6] builds on [22], but focuses on the privacy aspect instead of auction design. [24] proposes a new definition of group bid which achieves better auction performance and preserves all economic properties. So our evaluation also compares with [24].

There are also some spectrum double auction designs that are not based on TRUST. However, they are either not truthful or do not support general conflict graphs. For example, [20] proposes an online double sided spectrum auction where buyers can come and request for resource at different times. It assumes a complete conflict graph (i.e., everyone interferes with everyone else), which simplifies the competition pattern and disables spectrum reuse. [23] proposes a single unit double auction with discriminatory pricing, but their auction is not proven to be truthful. A few works consider practical issues in spectrum auction. [2] proposes a double auction similar to TRUST, but takes buyers' frequency preference into consideration while forming groups. This is complementary to our work. [21] focuses on incorporating market locality. In addition, [7] proposes a double auction based approach for mobile data offloading, but it does not consider wireless interference as in spectrum auctions.

\section{OUR SOLUTION}

\section{A. Overview}

Design strategy: We develop a novel double auction for dynamic spectrum allocation. It consists of the following three parts: (i) seller side auction design, assuming $N$ channels sell (ii) buyer side auction design, assuming $N$ channels sell, and (iii) a procedure to determine the number of channels $N$ to sell to satisfy budget balance.

To the best of our knowledge, our solution represents the first double auction design for spectrum allocation that explicitly decouples the buyer side and seller side auction design 
while achieving (i) truthfulness, (ii) individual rationality, and (iii) budget balance. Previously, seller and buyer side auctions were designed jointly in order to satisfy all three properties (e.g., McAfee's principle used in TRUST and all its variants).

Decoupling seller side and buyer side design is crucial for dynamic spectrum allocation for two reasons. First, using separate auction designs for the buyers and sellers, we no longer require groups of buyers that share the same channel to be formed in advance to match with sellers one by one. Instead we can make more informed decisions based on the bids to select a stronger set of winners and improve efficiency, revenue, and utilization. Second, it enables flexible combination of different buyer/seller side designs. This is especially beneficial in our context since the two sides have rather different properties. For example, it is commonly assumed that a seller can sell to any buyer in the auction. Competition between sellers is similar to traditional auctions, whereas the buyers' competition is much more complicated due to complicated wireless interference.

Proof strategy: The key to our ability to design two sides separately is a new proof strategy for establishing truthfulness in a (decoupled) double auction. Formally we have the following theorem:

Theorem 1: A double auction for dynamic spectrum allocation is truthful if the following two conditions hold: (i) both seller side and buyer side auctions are truthful when the number of channels that are sold, denoted as $N$, is fixed, and (ii) no seller or buyer can improve its own utility by unilaterally modifying its own bid and causing $N$ to change.

The correctness of this theorem is easy to see: When a bidder lies but does not change $N$, he cannot gain because both sides are truthful when $N$ is fixed. When a bidder lies and changes $N$, it can not gain either because no seller or buyer can improve its own utility by unilaterally modifying its own bid and causing $N$ to change. Thus a bidder never gains by lying and the auction is truthful.

We use the following theorem from [9] to assist our design:

Theorem 2: If every losing bidder (i.e., unallocated) in an auction pays nothing, it is truthful if and only if its allocation algorithm is monotonic and it uses critical value as payment.

Design overview: Below is an overview of our design. The buyer side auction is complicated since buyers' competition is determined by the complex conflict graph and buyers without interference can share a channel. Therefore this paper focuses on the buyer side auction design.

The seller side auction is a standard auction, since a seller can sell to any buyer in the auction. Therefore we can apply uniform pricing or VCG for single-unit auction. We prove it satisfies the properties specified in Theorem 1.

To determine the number of channels $N$, we start by setting $N$ as the total number of channels that sellers collectively have. We run seller and buyer side auctions separately, and then check if budget balance is satisfied (i.e., the payment collected from the buyers is no less than the payment to be paid to the sellers). Note the payment is determined by our seller side and buyer side auction design described below and not the sum of winning bids/asks. If budget balance is already satisfied, we terminate. Otherwise, we decrease $N$ by 1 , and run the auction again. By reducing $N$, it requires buyers to bid even higher to win and sellers to ask even lower, thus reducing the gap between revenue and payment. We stop when the budget balance is satisfied and $N$ channels are then traded. This procedure always terminates because budget balance is satisfied when $N$ drops to 0 . Thus we guarantee budget balance, which is formally stated in the following theorem.

\section{Theorem 3: Our design satisfies budget balance.}

Note that McAfee's design is a special case of our framework, where both the buyer side design and the seller side design use uniform pricing. This shows that our framework is general and under our framework it is possible to design a double auction that satisfies all three economic properties. Below we describe our design in detail.

\section{B. Seller side design}

The seller side design is a standard auction since there is no need to consider interference among sellers. Thus we simply use the traditional uniform price design. Assuming $N$ channels are sold, the $N$ sellers with the lowest asking prices win, and they each get paid at the $N+1$-th seller's asking price. This uniform price design is known to be truthful in a single unit auction when $N$ is fixed. Moreover, since sellers are paid higher than their asking price, individual rationality is satisfied. In Appendix, we further prove theorem 4 to ensure this design is truthful when applied to a double auction.

Theorem 4: This seller side design, when applied to double auctions, does not allow a seller to unilaterally manipulate $N$ (i.e., the number of channels that can be sold) and gain.

\section{Buyer side design}

Overview: To ensure fairness and achieve high revenue, it is important for the buyer side auction to explicitly take into account the fact that different buyers face different levels of competition depending on their locations and interference pattern. Existing approaches do not account for such individuality and apply uniform pricing, where all buyers that share the same channel pay the same amount. This is not only unfair, but also reduces revenue since a buyer's payment is limited by the lowest bids among the buyers that share the channel.

Our key idea is thus divide-and-conquer. Specifically, we first partition the conflict graph into subgraphs. Independent sets are then constructed within each subgraph and pricing is computed independently in each subgraph. We then design a merge strategy to combine the winners from different subgraphs while preserving truthfulness. Note that when computing the allocation within each individual subgraph, we ignore the inter-subgraph conflict edges. During the merge procedure, we need to add back the inter-subgraph conflict edges. As a result, some winners from individual subgraphs may have to be removed due to the inter-subgraph conflict edges. The challenge is how to do so without compromising truthfulness.

Benefit of graph partition: Before going to the details of our design, we first show the two major benefits of graph partition: (i) better independent set construction, and (ii) higher revenue due to the removal of the uniform pricing constraint. 


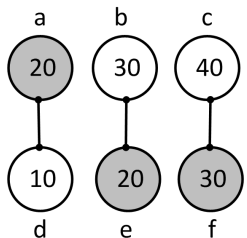

(a) Without partition

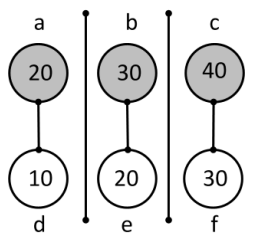

(b) With partition
Fig. 2. Benefit of graph partition

We illustrate these benefits using an example in Figure 2. It shows a conflict graph involving 6 nodes. We ignore the seller side in this example and assume we want to allocate 1 channel. To see the benefit (i), Figure 2(a) shows the groups constructed by TRUST: $G 1$ : $(a, e, f)$ and $G 2:(b, c, d)$. The group bids of $G 1$ and $G 2$ are $20 \times 3=60$ and $10 \times 3=30$, respectively, which are the lowest bid times the group size. So in TRUST $G 1$ wins and pays the first losing group's bid, which is 30 . The efficiency on the buyer side is the sum of all winning buyers' bids, which is $20+20+30=70$, and the revenue is 30 . Now if we treat each subgraph independently as shown in Figure 2(b): $a, b$ and $c$ win in their subgraphs and they each pays the first losing buyer's bid, which are 10, 20, and 30, respectively. Then we get the efficiency of $20+30+40=90$ and revenue of $10+20+30=60$, both of which are much higher than TRUST.

To demonstrate the benefit (ii), we consider TRUST happens to construct the same groups as ours, namely $G 1^{\prime}$ : ( $a$, $b, c)$ and $G 2^{\prime}:(d, e, f)$. The group bids of $G 1^{\prime}$ and $G 2^{\prime}$ are $20 \times 3=60$ and $10 \times 3=30$, respectively. So $G 1^{\prime}$ wins, and pays the first losing group's group bid, which is 30 and still lower than the revenue in our scheme, because the revenue of TRUST is limited by the uniform pricing (i.e., the lowest bid times the number of buyers). The difference in revenue may have even bigger impact when we take the seller side into consideration. If the revenue is lower than the amount sellers should get, some channels cannot be traded because it violates budget balance, which further reduces efficiency and revenue.

Essentially partitioning allows us to decouple nodes with no or weak interference into different subgraphs to improve the independent set construction and avoid unnecessary coupling in different buyers' pricing even though they interfere with different sets of nodes. Note the example shows a disconnected conflict graph but the idea also works on realistic, densely connected conflict graphs as shown in Section V.

Design questions: Several important questions should be addressed in order to realize the benefits of graph partition:

- How to partition the graph to retain important interference relationships within a subgraph and decouple nodes with weak and no interference into different subgraphs?

- How to compute auction results within each partition?

- How to merge the auction results from different partitions to achieve truthfulness and budget-balance?

Below we answer each question in turn.,

1) Graph partitioning: Given a graph $A$, a graph partitioning algorithm strives to find a partitioning $A_{1}, A_{2}, \ldots, A_{k}$ that minimizes a certain objective. For the purpose of dynamic spectrum allocation through double auction, a good graph partitioning algorithm should balance two key requirements: (i) the number of inter-subgraph edges should be small, and (ii) different subgraphs should be similar in size. If a subgraph is too small (e.g., having only one buyer), a small number of channels can satisfy all its buyers, leaving no losing buyers and no revenue from the subgraph. On the other hand, a too big subgraph may lead to poor independent set construction and poor performance due to the uniform pricing in each group.

Two common objectives have been proposed for graph partition: RatioCut and Normalized cut (NCut). The former normalizes the weights of the edges on the cut by the number of vertices in each partition (i.e., minimizes $\sum_{i=1}^{k} \frac{W\left(A_{i}, \overline{A_{i}}\right)}{\left|A_{i}\right|}$, where $W\left(A_{i}, \overline{A_{i}}\right)$ is the total weight of all edges between $A_{i}$ and the remaining nodes $\overline{A_{i}}$, and $\left|A_{i}\right|$ denotes the number of vertices in $A_{i}$ ). The latter normalizes the weights of the edges on the cut by the sum of node degrees in each partition (i.e., minimizes $\sum_{i=1}^{k} \frac{W\left(A_{i}, \overline{A_{i}}\right)}{\operatorname{vol}\left(A_{i}\right)}$, where $\operatorname{vol}\left(A_{i}\right)$ denotes the sum of degrees of all nodes in the partition $A_{i}$ ). It is easy to see $\frac{1}{\left|A_{i}\right|}$ and $\frac{1}{\operatorname{vol}\left(A_{i}\right)}$ are minimized when either the number of vertices or the sum of node degrees within each partition is the same. This captures our goal of finding balanced cuts while minimizing the weights of edges on the cut.

Minimizing either RatioCut or NCut is a NP-hard problem. Spectral clustering is a well-known effective scheme to find approximate solutions to this NP-hard problem (see [11] for a nice tutorial). There are many variants of spectral clustering [11], [13], [15]. In this paper, we use the Meila-Shi algorithm [13] to minimize NCut, which is the recommended algorithm in [11] due to its excellent performance and solid mathematical foundation. Let $W$ be the adjacency matrix with weight $w_{i j}$ on its $i$-th row and $j$-th column. Let $D$ be the degree matrix, which is a diagonal matrix with the node degree $d_{i}=\sum_{j} w_{i j}$ on the diagonal. The Meila-Shi algorithm takes the eigenvectors corresponding to the $k$ smallest eigenvalues of the normalized graph Laplacian matrix $L_{\mathrm{rw}}=I-D^{-1} W$ (where $I$ is the identity matrix) and then invokes another algorithm (e.g., k-means clustering [10]) to cluster points by their respective $k$ components in these eigenvectors.

To automatically determine the number of clusters to create (i.e., k), we follow the suggestion of [11] and apply the eigengap heuristic. Specifically, let $\lambda_{1} \leq \lambda_{2} \leq \cdots$ be the eigenvalues of the normalized graph Laplacian matrix $L_{\text {rw }}$ sorted in an ascending order. The eigengap heuristic computes all the eigengaps (i.e., difference between two successive eigenvalues) and chooses the number of clusters $k$ such that $\left(\lambda_{k+1}-\lambda_{k}\right)$ is the largest eigengap.

Note that when the conflict graph is disconnected, we first divide it into multiple connected components since nodes in different connected components have no competition at all. Then we apply spectral clustering to each connected component to further partition the connected component.

2) Allocation within a subgraph: When a good partitioning is found, we first compute the allocation in each subgraph independently. For that we can apply existing algorithms, such as TRUST. To further improve the performance, in our implementation we apply the allocation algorithm proposed in TDSA [24]. It is similar to TRUST but it defines a new group bid and allows a subset of a group to win while the rest 


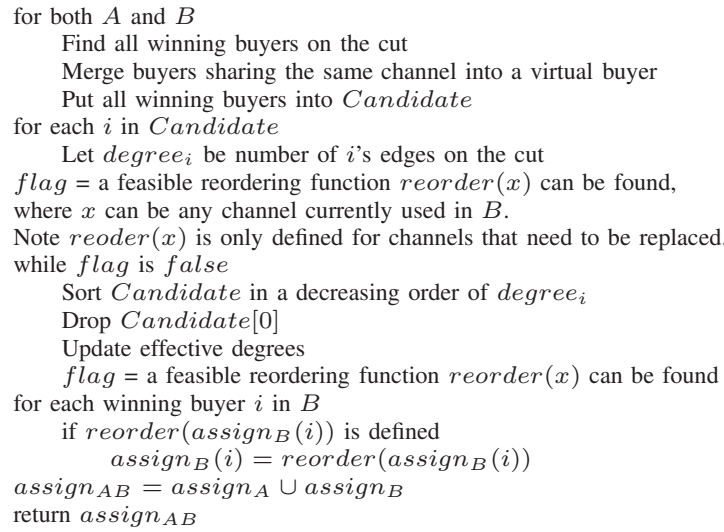

Fig. 3. Pseudo code for bid-independent merge.

lose. Consider a group $k$ and assume its members are sorted in a decreasing order of their bids. Denote members as 1 to $m$, and the group bid as $\max \left\{b_{i} \times i \mid i=1 \ldots m\right\}$. This group bid quantifies the maximum potential payment of a group if we allow a subset of this group to win. For each subset, the maximum potential payment is the lowest bid in the subset times the size of the subset. Thus this group bid finds the highest potential payment by enumerating all possible sizes of the subset. For example, if a group contains bids $(1,3,5)$, its potential payment could be either letting 5 win alone, which corresponds to payment of $5 \times 1=5$; or letting 3 and 5 win together, which yields a payment of $3 \times 2=6$; or letting all of them win, which yields a payment of $1 \times 3=3$. The maximum payment achieved is 6 in this example.

To allocate $N$ channels, the groups with the top $N$ group bids win. A winning group is then charged the first losing group's group bid and all members in the group share the price equally. If a member $i$ cannot afford its fair share (i.e., $b_{i} * i$ is smaller than the first losing group bid, which is possible due to TDSA-based group bid computation), $i$ does not win and the price is shared among the remaining group members. Since the winning group has a higher group bid than the losing group, there always exists a subset of the group such that they all bid no lower than their fair share. This automatically guarantees individual rationality on the buyer side. We prove that this procedure finds the critical value of a buyer under our partition and merge framework in Theorem 6 in the Appendix.

We also make a temporary assignment by sorting the channels in an increasing order of their asking prices and assign the first channel to the first group and second channel to the second group, and so on. However, this assignment is subject to change in the merge procedure below.

3) Merge strategy: Next we describe how to merge allocation results from different subgraphs. We merge two subgraphs at a time. The input of merge is the winners selected from the two subgraphs, including their channel assignment. The purpose of the merge is to find one way to reorder the channel assignment in one subgraph such that winners on the cut do not have conflict. If such reordering does not exist, certain nodes on the cut may be dropped. We propose the following bid-independent merge strategy to select a node to drop. We prove this merge strategy preserves truthfulness.

Figure 3 gives the pseudo-code of the merge procedure. In lines $1-4$, we preprocess the buyers on the cut by combining the buyers that share the same channel on each side into one virtual node, because these buyers are always assigned the same channel no matter how the assignment is reordered. Lines 5-6 compute the effective degree for each buyer $i$, which denotes the number of edges that $i$ has on the cut. This information is used in lines 7-12 to determine which buyer to drop when a feasible reordering does not exist. We search for a feasible reordering by enumerating all possibilities. The cost of this procedure is acceptable because its complexity depends on the number of channels (which is small) instead of number of buyers. We drop buyers in a decreasing order of their effective degrees in order to minimize the number of dropped buyers and reduce loss in efficiency and revenue. Every time a buyer is dropped, the effective degrees of the remaining nodes are updated. We iterate until a feasible reordering is found, and then use it to derive a joint assignment for the union of $A$ and $B$. Intuitively, our partition and merge framework preserves truthfulness because both operations by themselves are bidindependent and there is no incentive to lie. It is still possible to lie to change the allocation in subgraphs, which changes the input of the merge operation, but a buyer cannot gain this way because of the use of critical value pricing. We formally prove truthfulness in Theorem 5.

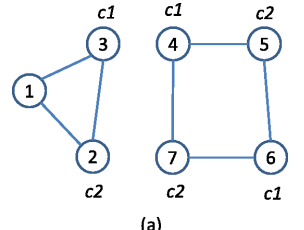

(a)

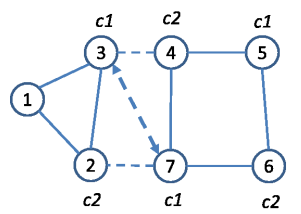

(c)
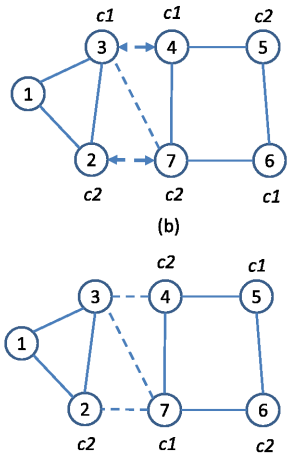

(d)
Fig. 4. A simple example of the merge procedure

Figure 4 shows an example of merging two subgraphs. Let $A$ and $B$ denote the left and right subgraphs, respectively, and $c 1$ and $c 2$ denote the channels to be allocated. The labels next to nodes represent the channel assignments to the nodes. Figure 4(a) shows the assignment to the winners in each subgraph. If in one subgraph, some winning buyers on the cut share the same channel, they should be merged to form a virtual buyer before we proceed. There are no such buyers in this case, so we go to the next step by adding back the removed edges. In Figure 4(b), the three previously removed edges are added back and there is conflict between nodes 3 and 4 and conflict between nodes 2 and 7. In Figure 4(c), the algorithm tries to reorder the assignment in $B$ by swapping $c 1$ and $c 2$ but there is still conflict between nodes 3 and 7 . So in Figure 4(d) node 3 , which is the node on the cut with highest degree, is dropped to resolve the conflict.

Theorem 5: $D A^{2}$ is truthful.

Proof: To prove this theorem, we first prove Theorems 6 and 7 in the Appendix, and then apply Theorems 1 and 4.

\section{Practical Issues}

Next we discuss practical issues involved in applying $D A^{2}$. 
Reputation score: The auctioneer maintains a reputation score $r_{i}\left(0<r_{i} \leq 1\right)$ for both buyers and sellers to reflect their quality. For a seller, the score can be based on his channel quality. For a buyer, it can be based on whether this buyer uses wireless resource carefully without causing extra interference to other buyers. The higher the score, the better the reputation. When computing the allocation, we divide every seller's asking price by its reputation score so that it is harder for a seller with a bad reputation to win. We then multiply a seller's reputation score with its critical value to compute its final payment. Since the critical value is computed based on the reputation weighted asking prices and is greater than a winner's asking price divided by its reputation, individual rationality is achieved. Similarly, for buyers we can multiply their bids by their reputations when computing the allocation, and then divide its critical value by its reputation score to get the real price they need to pay to ensure individual rationality. Truthfulness is preserved as the reputation scores do not depend on bids. Moreover, budget balance is still satisfied because we determine $N$ based on the the real selling price and payment (after multiplying/dividing the reputation scores).

Leveraging prior knowledge: The independent set construction is critical to the performance. Our solution mitigates the randomness but the construction can be further improved if the auctioneer has prior knowledge, e.g., distributions of buyers' valuations. Specifically, we can maximize the expected valuations in winning groups, which directly relates to auction efficiency by formulating the construction as a maximum weight independent set problem (MWIS). The expected valuation of an independent set is simply the sum of expected valuations of all the members. The MWIS problem on conflict graphs can be approximated in polynomial time, e.g., [16]. When the error of prior knowledge with respect to the actual bids is up to $10 \%$, the efficiency improves by $16 \%$ over our current scheme on average; and when the error increases to up to $50 \%$, the average improvement becomes $6 \%$.

Avoiding starvation: Our conflict graph partition is designed to minimize the number of inter-subgraph edges, while balancing the subgraphs. However in extreme cases it is still possible that well connected nodes are on the cut of two subgraphs. These nodes may consistently be dropped even if they win in their subgraph due to their large degrees and get starved. To address the starvation issue, we can introduce randomness in the merge procedure by dropping a buyer with a probability proportional to its degree. So well connected nodes are more likely to be dropped, but they are not always dropped and will not starve. This new procedure is still bid-independent, so truthfulness is preserved.

\section{Evaluation}

Simulation setup: In order to experiment with realistic conflict graphs, we use the location data of cell towers from a large US service provider. We consider there are buyers at each tower/location looking for spectrum resources at that location and that they do not collude. We construct the conflict graph for three cities: New York City (NYC), San Francisco (SF) and Chicago. In each city, we pick a grid (approximately $5 \mathrm{~km}$ by $5 \mathrm{~km}$ ) encompassing the downtown area and use all the cell towers in the grid to generate the conflict graphs. We consider that two nodes conflict if the inter-node distance is smaller than $500 \mathrm{~m}$, which is considered a typical cell range. We also vary the range to see how the network density impacts the performance. We only present results with conflict graphs generated based on real locations, but we also experimented with random conflict graphs and observed similar benefits.

We use 5 sellers by default, and also vary the number from 3 to 7 to see the impact. The small number of sellers is consistent with the reality where there are only a few large spectrum owners. The number of winning sellers is constrained by the conflict graph and increasing the number of sellers further has little impact on the result. For the sellers' asks and buyers' bids, the absolute values do not matter and only their ratio matters. We generate the buyers' bids drawn from a uniform distribution between 0 to 100 . We also use a uniform distribution to generate the asking prices. Since each grid can cover at most 25 buyers and we assume buyers and sellers value the spectrum resource similarly such that the price from the two sides compare fairly, we generate the asking prices to be between 0 to 2500 such that the mean is 25 times of the mean of a buyer's bid. We also scale the sellers' asking prices to see its impact in our evaluation.

We compare our scheme with TRUST and TDSA [24] in terms of the following three metrics: (i) Efficiency: This is widely used to quantify auction performance. It is defined as the difference between the sum of all winning buyers' bids and the sum of all winning sellers' asks. (ii) Revenue: It is defined as the total payment from all winning buyers. Revenue is different from efficiency on the buyer side since revenue depends on critical values whereas efficiency depends on bids. A higher revenue gives a stronger incentive for sellers to participate. (iii) Utilization: This is defined as the number of winning buyers. This is a unique metric in spectrum auctions because the spectrum resource is precious but reusable, and a higher utilization means more winners can utilize the spectrum at the same time, which is preferred. Utilization cannot be derived from efficiency since the winning buyers may not be the ones with the highest bids and it is impossible to tell the number of winning buyers just from the efficiency value. For every setting, we run 20 times with different random asking prices and bids, and report the average.

Performance at different locations: We first compare the performance in all three cities. As shown in Figure 5, the performance varies significantly across cities. Specifically, SF gives the highest values in all three metrics while Chicago gives the lowest and NYC lies in between. The performance difference is primarily due to difference in the number of buyers. SF has $16 \%$ more buyers than NYC, while the number of buyers in Chicago is only around half of that in NYC. In general, more buyers tend to generate higher revenue, thus more channels can be traded. Among all the schemes, $D A^{2}$ achieves the best performance in all three metrics and for all three cities. It improves efficiency to $22 \mathrm{x}$ to $62 \mathrm{x}$ of that of TRUST, revenue $27 \mathrm{x}$ to $126 \mathrm{x}$, utilization $42 \mathrm{x}$ to $65 \mathrm{x}$. TRUST does not perform as well because it is limited by the uniform pricing and the lowest bid in each group. As a result, its revenue is low and only few channels can be sold. TDSA performs better than TRUST because it searches for the best subset of a group to win and is thus more robust to the lowest bid in a group. $D A^{2}$ still outperforms TDSA by $51 \%$ to $101 \%$ 
in efficiency, $57 \%$ to $115 \%$ in revenue, and $47 \%$ to $93 \%$ in utilization. This is because $D A^{2}$ (i) decouples pricing in different subgraphs to better capture the competition, and (ii) combines the top groups from different subgraphs to reduce randomness and find a set of winners with higher valuations.

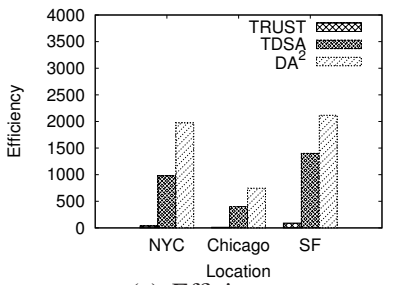

(a) Efficiency

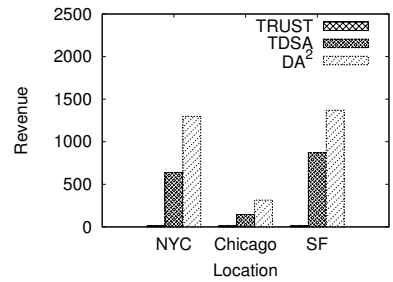

(b) Revenue

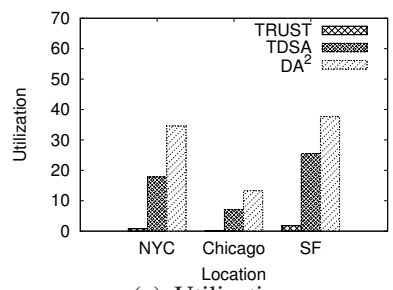

(c) Utilization

Fig. 5. Performance at different locations

In the remaining evaluation, we use the NYC conflict graph as default and study the impact of other parameters.

Impact of the number of sellers: Next we vary the number of sellers. Figure 6 shows that as the number of sellers increases, more channels become available and the price reduces due to increased seller-side competition. As a result, more channels can be traded and all performance metrics improve. $D A^{2}$ consistently out-performs the existing approaches. Its improvement is highest when there are only 3 sellers, in which case they achieve as much as $3 x$ times the performance of TDSA in all three metrics. TRUST does not sell a single channel in the 20 runs with 3 sellers because its revenue is low and it is more challenging to sell a channel while ensuring budget balance when the number of sellers is small. When the number of sellers increases to $7, D A^{2}$ out-performs TRUST by $27 \mathrm{x}$ in efficiency, $71 \mathrm{x}$ in revenue, and $23 \mathrm{x}$ in utilization; and outperforms TDSA by $85 \%$ in efficiency, $97 \%$ in revenue, and $86 \%$ in utilization.

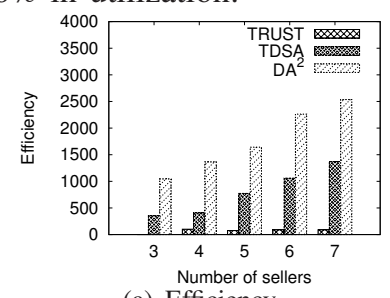

(a) Efficiency

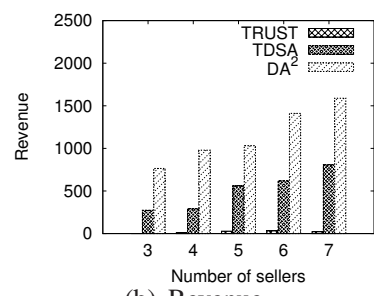

(b) Revenue

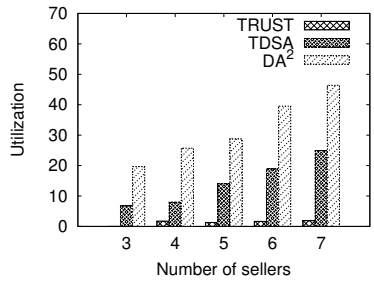

(c) Utilization

Fig. 6. Impact of the number of sellers

Impact of network density: Next we vary the network density by changing the buyer communication range from $250 \mathrm{~m}$ to $750 \mathrm{~m}$. A longer range indicates more competition among buyers and fewer buyers can reuse a channel. As shown in Figure 7, the benefit of our scheme increases with the range since it is harder to sell a channel and a good auction design becomes even more important. For example, when the range is $250 \mathrm{~m}$, our scheme out-performs TDSA by $29 \%$ in efficiency, $55 \%$ in revenue, and $30 \%$ in utilization. The corresponding numbers for a range of $750 \mathrm{~m}$ are $152 \%, 172 \%$ and $173 \%$. The performance trend in TRUST is less clear because TRUST heavily depends on the random independent set construction. However, under all three ranges, $D A^{2}$ achieves $14 x$ the efficiency, $22 \mathrm{x}$ the revenue, and $13 \mathrm{x}$ the utilization of TRUST.

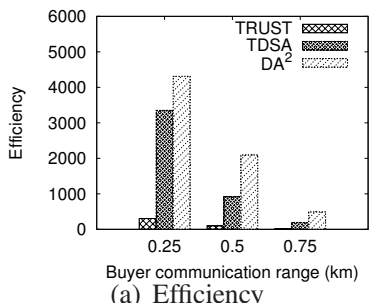

(a) Efficiency

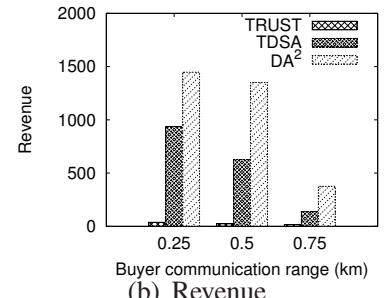

(b) Revenue

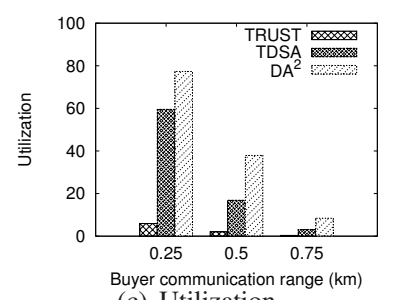

(c) Utilization

Fig. 7. Impact of network density

Impact of bid distribution: To understand the impact of bid distribution, we scale all the sellers' asking prices by a factor between 0.5 to 1.5 after the initial ask is drawn from a uniform distribution between 0 and 2500. This changes the ratios between sellers' valuation and buyers' valuation. A higher asking price requires a higher revenue from the buyer side in order to sell the channels, and is more challenging for an auction. This trend is shown in Figure 8. Moreover, in all cases $D A^{2}$ outperforms the existing schemes. The benefit of $D A^{2}$ increases with the asking price. For example, when we scale the asking price to 1.5 times, our approach out-performs TDSA by 3 times in all three metrics. The improvement over TRUST is even larger, as TRUST does not sell anything and all its metrics are 0 .

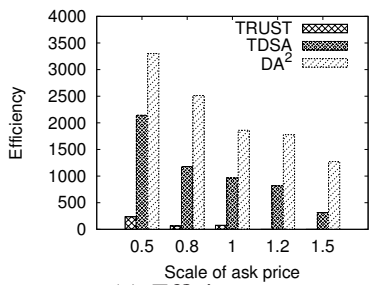

(a) Efficiency

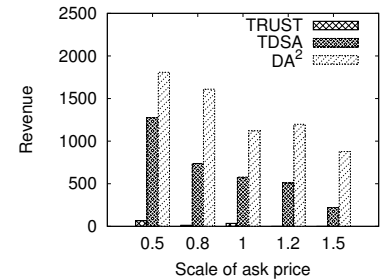

(b) Revenue

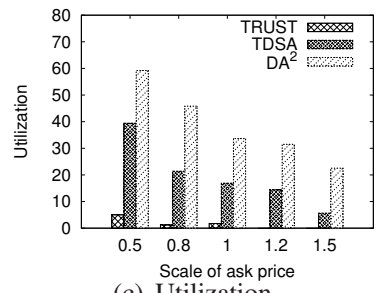

(c) Utilization

Fig. 8. Impact of bid distribution 


\section{CONCLUSION}

In this paper, we develop a truthful spectrum double auction to allow different providers to buy and sell spectrum to each other dynamically. We explicitly decouple buyer side design and seller side design to capture the different properties of the two sides. To overcome the challenges due to complex interference patterns on the buyer side, we partition the conflict graph to decouple nodes with no or weak interference while putting nodes with strong interference in the same subgraph so that group bids and critical values can accurately capture the real competition among different buyers. We design a novel merge strategy that combines the auction results from different subgraphs to achieve truthfulness. Using real cell tower topology traces from a large US wireless provider, we show that our approach achieves high efficiency, revenue, and fairness, and significantly out-performs the existing approaches.

Acknowledgements: This work is supported in part by NSF Grants CNS-1017549 and CNS-1343383.

\section{REFERENCES}

[1] N. Ahmed and S. Keshav. SMARTA: a self-managing architecture for thin access points. In Proc. of CoNEXT, 2006.

[2] X. Feng, Y. Chen, J. Zhang, Q. Zhang, and B. Li. TAHES: a truthful double auction mechanism for heterogeneous spectrums. Wireless Communications, IEEE Transactions on, 11(11):4038 -4047, Nov. 2012.

[3] Handover. http://xa.yimg.com/kq/groups/19564168/454394754/name/ 05-Handover.pdf.

[4] Handover tutorial. http://www.radio-electronics.com/info/ cellulartelecomms/gsm_technical/h\%andover-handoff.php.

[5] S. S. Hong, J. Mehlman, and S. Katti. Picasso: flexible RF and spectrum slicing. In Proc. of ACM SIGCOMM, 2012.

[6] Q. Huang, Y. Tao, and F. Wu. Spring: A strategy-proof and privacy preserving spectrum auction mechanism. In IEEE INFOCOM, 2013.

[7] G. Iosifidis, L. Gao, J. Huang, and L. Tassiulas. An iterative double auction for mobile data offloading. IEEE WiOpt, 2013.

[8] K. Jain, J. Padhye, V. N. Padmanabhan, and L. Qiu. Impact of interference on multi-hop wireless network performance. In Proc. of ACM MobiCom, 2003.

[9] A. Lapets and A. Levin. Restricted truthful combinatorial auction mechanisms, 2007

[10] S. Lloyd. Least squares quantization in PCM. IEEE Transactions on Information Theory, 28(2):129 - 137, 1982

[11] U. V. Luxburg. A tutorial on spectral clustering. Statistics and Computing, 2007.

[12] R. P. McAfee. A dominant strategy double auction. Journal of Economic Theory, 56(2):434-450, April 1992.

[13] M. Meila and J. Shi. A random walk view of image segmentation. In Proc. of AI and STATISTICS (AISTATS), 2001.

[14] R. B. Myerson and M. A. Satterthwaite. Efficient mechanisms for bilateral trading. Journal of Economic Theory, 29(2):265-281, 1983.

[15] A. Y. Ng, M. I. Jordan, and Y. Weiss. On spectral clustering: Analysis and an algorithm. In Proc. of NIPS, 2001.

[16] T. Nieberg, J. Hurink, and W. Kern. A robust PTAS for maximum weight independent sets in unit disk graphs. In Proc. of International Conference on Graph-Theoretic Concepts in Computer Science, 2004.

[17] J. Padhye, S. Agarwal, V. N. Padmanabhan, L. Qiu, A. Rao, and B. Zill Estimation of link interference in static multi-hop wireless networks. In Proc. of ACM IMC, 2005.

[18] V. Shrivastava, S. Rayanchu, S. Banerjee, and K. Papagiannaki. Pie in the sky: Online passive interference estimation for enterprise WLANs. In Proc. of NSDI, 2011.

[19] K. Tan, H. Shen, J. Zhang, and Y. Zhang. Enable flexible spectrum access with spectrum virtualization. In Proc. of Dyspan, Sept. 2012.

[20] S. Wang, P. Xu, X. Xu, S. Tang, X. Li, and X. Liu. TODA: truthful online double auction for spectrum allocation in wireless networks. In Proc. of New Frontiers in Dynamic Spectrum, pages 1 -10, Apr. 2010.

[21] W. Wang, B. Li, and B. Liang. District: Embracing local markets in truthful spectrum double auctions. In Proc. of IEEE SECON, 2011.
[22] F. Wu and N. Vaidya. Small: A strategy-proof mechanism for radio spectrum allocation. In Proc. of IEEE INFOCOM, Apr. 2011.

[23] L. Xiang, G. Sun, J. Liu, X. Wang, and L. Li. A discriminatory pricing double auction for spectrum allocation. In Proc. of WCNC, Apr. 2012.

[24] E. Yao, L. Lu, and W. Jiang. An efficient truthful double spectrum auction design for dynamic spectrum access. In Proc. of CROWNCOM, Jun. 2011.

[25] S. Yun, D. Kim, and L. Qiu. Fine-grained spectrum adaptation in wifi networks. In Proc. of ACM MobiCom, Sept. 2013.

[26] X. Zhou, Z. Zhang, G. Wang, X. Yu, B. Y. Zhao, and H. Zheng. Practica conflict graphs for dynamic spectrum distribution. In In Proc. of ACM SIGMETRICS, pages 5-16, New York, NY, USA, 2013. ACM.

[27] X. Zhou and H. Zheng. Trust: A general framework for truthful double spectrum auctions. In Proc. of IEEE INFOCOM, 2009.

\section{APPENDIX}

Proof of Theorem 4

Proof: To change $N$ by lying, a seller $i$ needs to change the point where the budget balance is satisfied, which requires the seller to change the total selling price. Let $S(N)$ be the total selling price when $N$ channels sell and $R(N)$ be the total revenue. Let $U_{i}(x, N)$ be the utility of seller $i$ when it asks $x$ and $N$ channels sell. Since the seller side design is truthful, we have $U_{i}\left(v_{i}, N\right) \geq U_{i}(x, N)$, where $v_{i}$ is $i$ 's true valuation and $x$ can be any value. We consider the following cases:

(1) $i$ is a winning seller: $i$ cannot change $N$ as long as it still wins. If it changes $N$ and now loses, its utility does not improve.

(2) $i$ is a losing seller. We consider the following cases:

- Lie by asking higher. Obviously, $N$ cannot increase in this case, since $S(N)$ does not reduce for any $N . i$ is a losing seller in the original case so it is not within the top $N . i$ cannot become the top $N$ by asking higher.

- Lie by asking lower. We only consider when $i$ becomes a winner, because its utility is still 0 if it still loses. We consider the following cases.

- Case 1: $N$ does not change: i's utility cannot improve because $U_{i}\left(v_{i}, N\right) \geq U_{i}(x, N)$.

- Case 2: $N$ reduces to $M: i$ is not in the top $M$ when it bids truthfully, so $U_{i}\left(v_{i}, M\right)=0$. Thus $i$ either receive 0 utility or negative utility because $U_{i}\left(v_{i}, M\right) \geq U_{i}(x, M)$

- Case 3: $N$ increases to $M$. We consider the following cases: $i$ is in the top $M$ when it bids truthfully, and the $M+1$-th asking price does not change. So $S(M)$ does not change. In the original case, only $N$ channels sell, so we know $S(M)>R(M)$. So budget balance is not satisfied and this case cannot happen.

$i$ is not among the top $M$ sellers when it asks truthfully. So $U_{i}\left(v_{i}, M\right)=0$. $i$ 's utility cannot increase in this case because $U_{i}\left(v_{i}, M\right) \geq U_{i}(x, M)$

Theorem 6: Our buyer side design is truthful when $N$ is fixed.

Proof: It is easy to see that the design is monotonic, i.e., if a winner wins at $v$, it still wins if it bids $b>v$, as bidding higher does not reduce the group bid and all other decisions are bid-independent. Next we verify that the price we charge is the critical value. Specifically we charge a winner the first losing group bid in its subgraph divided by $k$, if $k$ members of its group bid higher than that. We find the maximum $k$, so no more members can be admitted and still afford the fair share. To prove it is the critical value, we should show (1) a buyer still wins if it bids higher, and (2) a buyer loses if it bids lower. (1) holds because the buyer's group bid (which is greater than $k$ times of the critical value) is still higher than the first losing group and the merge process is bid-independent. To see (2), we consider three cases: (i) $k$ remains the same, (ii) $k$ increases, and (iii) $k$ decreases. For (i), if a buyer bids lower than that value, it cannot afford its fair share for the same $k$ even if its group still wins. (ii) cannot happen because other members' bids do not change and no new members can afford the fair share. In (iii), the fair share is even larger as $k$ decreases, so the buyer does not win either. Thus a buyer never wins if it bids lower than the price we charge. Therefore the buyer side with bid-independent merge is truthful when $N$ is fixed according to Theorem 2.

Theorem 7: Bid-independent merge based allocation does not allow a buyer to unilaterally change $N$ and gain.

Proof: The proof has same structure as the proof to Theorem 4 but from a buyer side. 